\title{
Antiangiogenic Therapeutic Potential of Peptides Derived from the Molecular Motor KIF13B that Transports VEGFR2 to Plasmalemma in Endothelial Cells
}

\author{
Kaori H. Yamada, ${ }^{* \dagger}$ Hojin Kang, ${ }^{* \dagger}$ and Asrar B. Malik ${ }^{* \dagger}$
}

From the Department of Pharmacology* and Center of Lung and Vascular Biology, ${ }^{\dagger}$ The University of Illinois College of Medicine, Chicago, Illinois

Accepted for publication September 15, 2016.

Address correspondence to Kaori H. Yamada, Ph.D., or Asrar B. Malik, Ph.D., E403 MSB, 835 S., Wolcott Ave., Chicago, IL 60612. E-mail: horiguch@uic.edu or abmalik@ uic.edu.

\begin{abstract}
Vascular endothelial growth factor receptor 2 (VEGFR2) localized on the surface of endothelial cells (ECS) is a key determinant of the magnitude and duration of angiogenesis induced by vascular endothelial growth factor (VEGF). The kinesin family plus-end motor KIF13B transports VEGFR2 to the EC surface, and as such, specific inhibition of polarized VEGFR2 trafficking prevents angiogenesis. We designed a series of bioactive peptides based on deep analysis of VEGFR2-binding domain of KIF13B that compete specifically with VEGFR2 binding of KIF13B and thereby potently inhibit angiogenesis. Expression of these peptides by lentivirus prevents VEGF-induced capillary network formation in Matrigel plugs and neovascularization in vivo. A synthetic soluble, cell-permeable, 23-amino acid peptide termed kinesin-derived angiogenesis inhibitor (KAI) not only prevents interaction of VEGFR2 with KIF13B but also trafficking of VEGFR2 in the plus-end direction to the EC plasmalemma. Kinesinderived angiogenesis inhibitor also inhibits VEGF-induced EC migration and tumor growth in human lung carcinoma xenografted in immunodeficient mice. Thus, we describe a novel class of peptides derived from the site of interaction of KIF13B with VEGFR2 that inhibit VEGFR2 trafficking and thereby starve cancer of blood supply. (Am J Pathol 2017, 187: 214-224; http://dx.doi.org/10.1016/ j.ajpath.2016.09.010)
\end{abstract}

Vascular endothelial growth factor (VEGF) plays a critical role in the metastasis and growth of cancer by increasing nutrient blood supply secondary to formation of new blood vessels. $^{1-3}$ VEGF signals through activation of the endothelial cell (EC) surface-localized high-affinity tyrosine kinase receptor VEGF receptor 2 (VEGFR2) (alias Flk-1). ${ }^{1}$ Poor vessel growth and maintenance cause ischemia in myocardial infarction, stroke, and neurodegenerative- and obesity-associated disorders, whereas excessive vascular growth is essential for metastasis and growth of cancer, inflammatory disorders, and retinal vascular diseases associated with diabetes and macular degeneration. ${ }^{2-5}$ Thus, angiogenesis is a key therapeutic target for these diseases. Anti-VEGF antibodies and kinase inhibitors ${ }^{7}$ prevented VEGFR2 signaling and EC migration. ${ }^{1-3}$ VEGF blockade also prolonged survival in monotherapy or combined therapy in patients with cancer ${ }^{7}$; however, the responses are variable, and some patients were refractory or in time acquired resistance. ${ }^{8}$ The mechanisms of resistance and variability have been discussed elsewhere. ${ }^{5,8-12}$

VEGF $^{165}$ binding to VEGFR2 induces VEGFR2 homodimerization, autophosphorylation, and internalization of VEGFR2. ${ }^{1}$ The internalized VEGFR2 is ubiquitinated and degraded in lysosomes. ${ }^{13}$ VEGF in addition to ligating the receptor signals the polarized trafficking of newly synthesized VEGFR2 from Golgi apparatus to the plasma membrane to continuously restore the cell surface receptor pool for the next round of VEGF binding and receptor activation

Supported by NIH grants PPG HL060678, R01 HL045638, P01 HL077806, R01 HL090152, R01 HL118068, and R01 HL125350 (A.B.M.), NIH grant R56 HL128342 (K.H.Y.), and American Heart Association grant 13SDG14680053 (K.H.Y.).

Disclosures: None declared.

A provisional patent has been filed regarding this peptide (2016-049-01) The patent covers peptide KAI for angiogenesis-related diseases, such as cancer and ocular diseases. 
cycle. ${ }^{14-16}$ This is evident by findings that inhibition of VEGFR2 trafficking prevented VEGF-mediated neovascularization in ears of mice ${ }^{14}$ and vessel formation in Matrigel plugs in vivo. ${ }^{16}$ The requirement for transport of VEGFR2 to the EC surface is especially important in the outward sprouting tip ECs, ${ }^{17}$ which form filopodia as the vessels grows outward while the stalk ECs just below the tip cells undergo increased proliferation in response to VEGF. ${ }^{18}$ Thus, VEGFR2 localization in tip cells is essential for directional migration of ECs in newly forming vessels. ${ }^{18}$ We recently found that KIF13B, a kinesin family motor, unidirectionally transported VEGFR2 along microtubules in response to VEGF. ${ }^{16}$ Furthermore, we found that inhibition of VEGFR2 trafficking by depletion of KIF13B abrogated EC migration and sprouting angiogenesis; however, EC survival and proliferation were unaffected. ${ }^{16}$ The tail of KIF13B has multiple domains for cargo binding. ${ }^{19-23}$ Besides VEGFR2, KIF13B transports polarity determination factors, such as PIP3. ${ }^{20,24}$ These cargoes bind distinct regions of KIF13B often by bridge proteins, such as centaurin- $\alpha .{ }^{20}$ This prompted us in the present study to target specifically VEGFR2 trafficking that would only prevent VEGF-dependent angiogenesis. We describe the minimal binding domain required for KIF13B interaction with VEGFR2 and define the role of peptides derived from this domain in modulating VEGFR2 trafficking and their therapeutic potential.

\section{Materials and Methods}

\section{Antibodies and Reagents}

Antibodies against KIF13B (Sigma, St. Louis, MO), extracellular domain of VEGFR2 (Fitzgerald, Acton, MA), E-tag (Abcam, Cambridge, MA), von Willebrand factor based on analysis of VEGFR2-binding site in KIF13B (Millipore, Temecula, CA), and CD31 (Abcam) were used. Secondary antibodies were horse radish peroxidase-conjugated donkey anti-rabbit (Jackson ImmunoResearch, West Grove, PA), Alexa594-conjugated anti-rabbit (Life Technologies, Carlsbad, CA). WST-1 (Roche, Basel, Switzerland) and terminal deoxynucleotidyl transferase-mediated dUTP nickend labeling (TUNEL) kit (Life Technologies) were also used.

\section{Plasmids}

Human KIF13B cDNA was purchased from the Kazusa DNA Institute (Chiba, Japan). ${ }^{16}$ Truncated mutants, lentivirus, and recombinant proteins were produced as described by Yamada et al. ${ }^{16}$

\section{Peptides}

Peptides were custom synthesized by Pierce Biotechnology (Rockford, IL) at 95\% purity, which was confirmed by reverse-phase high-performance liquid chromatography and mass spectrophotometry. Bulk peptides were aliquoted in glass vials $(25 \mathrm{mg}$ per vial) and kept in a desiccator at $-20^{\circ} \mathrm{C}$. Once dissolved in sterile water in acidic condition (by adding $<10 \%$ sterile acetic acid), $\mathrm{pH}$ was adjusted by adding sterile phosphate-buffered saline (PBS), and $10 \mathrm{mg}$ / $\mathrm{mL}$ of the peptide in sterile PBS was aliquoted and kept at $-20^{\circ} \mathrm{C}$ freezer. Once thawed, peptide solutions were used within 2 to 3 days.

\section{Cell Culture}

Human primary umbilical vein endothelial cells (HUVECs) (Lonza, Walkersville, NJ), HEK293T/17, and human fibroblast Detroit 551 (ATCC, Manassas, VA) were maintained as described by Yamada et al. ${ }^{16} \mathrm{H} 460$ cells were from ATCC and maintained in Dulbecco's modified Eagle's medium (Life Technologies) supplemented with $10 \%$ fetal bovine serum.

\section{Angiogenesis Assays}

Collagen invasion assay was performed as previously described. ${ }^{25}$ In vitro capillary network formation on Matrigel (BD Biosciences, San Jose, CA) and scratch wound healing assay were performed as previously described. ${ }^{16}$ Fibrin gel sprouting assay was performed as described by Nakatsu and Hughes. ${ }^{26}$ VEGF $(2.2 \mathrm{nmol} / \mathrm{L})$ stimulation was used for all in vitro angiogenesis assays. Transwell migration assay was performed as described by Kaplan et al, ${ }^{27}$ using VEGF (4.4 nmol/L), sphingosine-1-phosphate (S1P) $(1 \mu \mathrm{mol} / \mathrm{L})$, or basic fibroblast growth factor (bFGF) (50 ng/ $\mathrm{mL}$ ) in the presence or absence of kinesin-derived angiogenesis inhibitor (KAI) at varying concentrations.

\section{In Vitro Binding}

Truncated mutants of KIF13B were expressed in BL21 (DE3) or Rosetta-gami and purified as previously described. ${ }^{16,19}$ Binding assay was performed with S-resin in $0.5 \%$ Triton-X100 and $1 \%$ bovine serum albumin at $4{ }^{\circ} \mathrm{C}$ and analyzed by Western blotting using anti-VEGFR2 antibody.

\section{Proliferation, Viability, and Toxicity Assays}

VEGF-induced proliferation and viability of HUVECs were assessed by the WST-1 assay (Roche) as previously described. ${ }^{28,29}$ HUVECs were cultured in reduced serum media (EBM2 supplemented with $0.1 \%$ fetal bovine serum and EC supplements, except VEGF, epidermal growth factor, insulin-like growth factor, and fibroblast growth factor). Then HUVECs were stimulated with VEGF $(2.2 \mathrm{nmol} / \mathrm{L})$, with and without $\mathrm{KAI}(1,3$, and $10 \mu \mathrm{mol} / \mathrm{L})$, or negative control peptide (CT23, $3 \mu \mathrm{mol} / \mathrm{L}$ ) for 48 hours to test VEGF-induced proliferation by WST-1 assay in a 96-well 
format. Viability of HUVECs was assessed with and without $\mathrm{KAI}(1,3$, and $10 \mu \mathrm{mol} / \mathrm{L})$ after overnight incubation in growth medium. Apoptosis was measured by TUNEL assay (Invitrogen) after incubation with and without KAI in growth medium for 48 hours. As a positive control, $50 \mathrm{ng} / \mathrm{mL}$ of tumor necrosis factor (TNF)- $\alpha$ was also used in the apoptosis assay.

\section{Endothelial Permeability Assay}

A VEGF-induced increase in endothelial permeability was assessed by leakage of fluorescein isothiocyanate-dextran across HUVEC monolayers. Transwells (with 0.4- $\mu$ m-pore diameters) were coated with confluent HUVECs. After serum starvation, cells were pretreated with KAI or CT23 $(10 \mu \mathrm{mol} / \mathrm{L})$. Then the cells were stimulated with VEGF, and at various time points transendothelial fluorescein isothiocyanate-dextran permeability was measured using the fluorescence plate reader PHERAstar (BMG Biotech, Cary, $\mathrm{NC})$.

\section{Matrigel Plug Vessel Formation Assay and Mouse Xenograft Model}

For the Matrigel plug assay used to assess vessel formation of mouse ECs, C57BL6 males (Jackson Laboratory) were used as previously described. ${ }^{16,30}$ For mouse xenograft model, human lung carcinoma $\mathrm{H} 460\left(3 \times 10^{6}\right.$ cells $)$ s.c. was inoculated in the right flank of immunodeficient BALBc mice with severe combined immunodeficiency (Jackson Laboratory) under anesthesia as described. ${ }^{31,32}$ After a palpable tumor had developed, mice received either $200 \mu \mathrm{L}$ of PBS or peptide KAI $(10 \mathrm{mg} / \mathrm{kg}$ dissolved in $200 \mu \mathrm{L}$ of PBS) by i.v. injection via tail vein 3 times per week. Tumor size was measured 3 times per week using a caliper. After the treatment period, the tumor was fixed and paraffin embedded for immunohistologic analysis with antiVEGFR2 antibody for assessment of tumor angiogenesis. The mice were housed in pathogen-free conditions in the University of Illinois Animal Care facility and treated in accordance with institutional guidelines.

\section{Postnatal Retina Angiogenesis}

Neonatal C57B mice were given peptide (either KAI or CT23, $10 \mathrm{mg} / \mathrm{kg}$ dissolved in PBS) or PBS vehicle at the day of birth (postnasal day 0) and subsequent days (postnasal days 1 to 4 ) daily s.c. under eyelid as previously described. ${ }^{33}$ At postnatal day 6 , the retina was isolated and stained with anti-CD31 antibody (Abcam) as previously described. $^{34}$

\section{Image Acquisition Protocol}

Microscopes used for this study were Zeiss confocal LSM 880 META with $63 \times$ oil immersion objective lens and Zeiss
Axiovert phase-contrast microscope with Plan-Neofluar $5 \times$ objective, $20 \times$ objective lens, and $40 \times$ oil immersion objective lens. All fluorescence images were taken in the same conditions and settings for all samples in the same set of experiments.

\section{Statistical Analysis}

The $t$-test was used for intergroup comparisons. To analyze more than two groups, one-way analysis of variance and post hoc Bonferroni multiple comparisons were performed with GraphPad Prism software version 5 (GraphPad Software, San Diego, CA).

\section{Results}

\section{Identification of Minimal KIF13B-Derived Peptide-} Inhibiting Angiogenesis

We used recombinant proteins to identify the binding sites on KIF13B mediating the interaction with VEGFR2. KIF13B has a motor, forkhead-associated, membrane-associated guanylate kinase-binding stalk, 2 domains of unknown function (DUFs) (DUF3694), and a proline-rich and cytoskeleton-associated protein glycine-rich domain (Figure 1A). The DUFs were identified from amino acid sequence similarity on the Pfam database (http://pfam.xfam. org/family/DUF3694, last accessed August 28, 2016). We previously found that both domains of DUF3694 directly bind recombinant VEGFR2 in vitro. ${ }^{16}$ To define the specific peptide sequence inhibiting VEGFR2 trafficking, we focused on the second DUF3694 domain (DUF2, 1112-1281 amino acids). This was based on stability of this domain and a sequence distinct from binding sites for other cargoes of KIF13B. ${ }^{20,21}$ We truncated DUF2 into 3 parts (A, B, C) and expressed these as recombinant proteins in bacteria. VEGFR2 was abundantly expressed in HUVECs; thus, cell lysate from HUVECs was incubated with these recombinant proteins on beads, and VEGFR2 binding was determined using the anti-VEGFR2 antibody (Figure 1B). We observed that DUF2C specifically binds VEGFR2, whereas DUF2A and $\mathrm{B}$ and beads alone failed to bind (Figure 1B).

To test the bioactivity of the truncated mutant of KIF13B, we expressed DUF2C by lentivirus vector in HUVECs. Expression of DUF2C was sufficient to reduce VEGFinduced capillary network formation in vitro, whereas vector-infected control HUVECs formed characteristic capillary networks in Matrigel plugs in the presence of VEGF (Figure 1C). We also used the collagen invasion system $^{25}$ as another angiogenesis assay to test the peptides. After infection with control lentivirus, FLAG-DUF2C, or FLAG-DUF2, HUVECs were seeded on collagen gels. Cell invasion in response to proangiogenic stimuli S1P and VEGF in the gel was monitored for up to 24 hours (Figure 1, D and E, and Supplemental Figure S1). Only the expression of DUF2C or the entire DUF2 sequence 
A
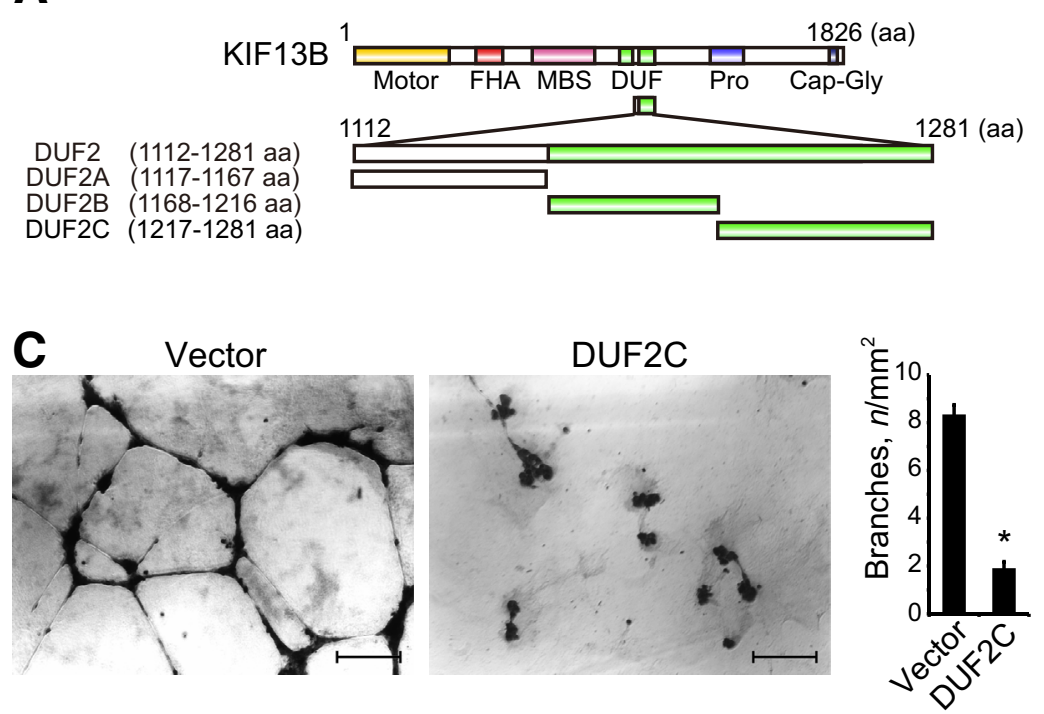

$\mathbf{E}$
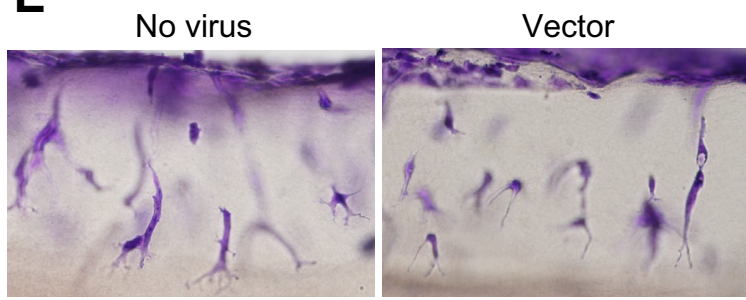

B
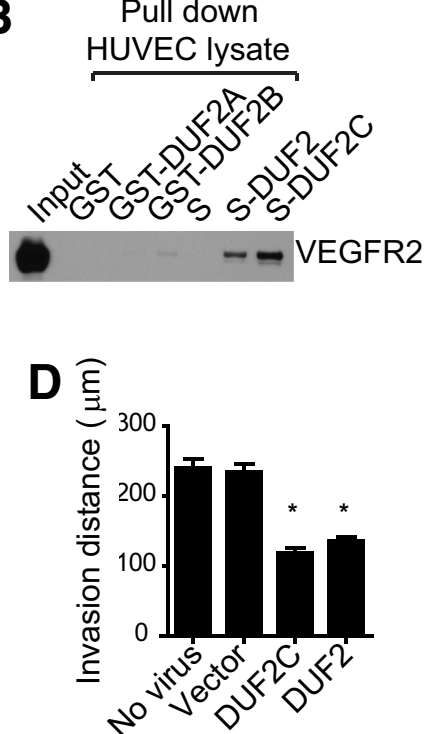

Figure 1 Identification of KIF13B domains inhibiting the interaction of KIF13B with vascular endothelial growth factor receptor 2 (VEGFR2). A: Schematic of the domains of KIF13B and truncated domains used in the study. B: For the in vitro binding assay, the domains of KIF13B [domains of unknown function (DUFs) 2A, 2B, and 2C] were expressed in bacteria and tested for binding to VEGFR2 in human primary umbilical vein endothelial cell (HUVEC) lysates by the pull-down assay. VEGFR2 was detected by Western blotting using an antibody. C: In vitro two-dimensional capillary network formation of HUVECs treated with either control virus (FLAG vector) or virus encoding truncated mutants of FLAG-KIF13B in Matrigel supplemented with VEGF (2.2 nmol/L). D and E: Vascular endothelial growth factor (VEGF) - and sphingosine-1-phosphate (S1P)-induced invasion in three-dimensional collagen matrices. HUVECS were infected without virus or with control versus FLAG-DUF2C or FLAG-DUF2 for 2 days and tested for invasion into collagen in the presence of S1P or VEGF (2.2 nmol/L). Results are representative of three individual experiments. Quantification of the collagen invasion assay is given in $\mathbf{D}$ by measuring distance of invading endothelial cells. Data are expressed as means \pm SEM (C and D). $n=15$ and 9 (C); $n=50$ sprouts (D). ${ }^{*} P<0.05$. Scale bars: $200 \mu \mathrm{m}(\mathbf{C}) ; 100 \mu \mathrm{m}$ (D). aa, amino acid; Cap-Gly, cytoskeleton-associated protein glycine-rich; FHA, forkhead associated; GST, glutathione $S$ transferase; MBS, membrane-associated guanylate kinase-binding stalk; pro, proline rich; S, oligopeptide derived from RNase A (alias S-tag).

significantly reduced invasion of HUVECs into collagen gel (Supplemental Figure S1), distance of invasion (Figure 1D and Supplemental Figure S1), and number of lumen formed (Supplemental Figure S1). Thickness of invading sprouts, however, was not different among the groups (Supplemental Figure S1).

We then followed up with further truncating DUF2C (DUF2C1-9) to identify the minimum binding site on VEGFR2 (Figure 2A). Among these, we identified the core binding region in 1238 to 1260 amino acids of KIF13B by pull-down binding assay with endogenous VEGFR2 from HUVEC lysates (Supplemental Figure S2, A-C) and termed it DUF2C5 (shorter name, C5) (Figure 2A). The truncated proteins $\mathrm{C} 6, \mathrm{C} 8$, and $\mathrm{C} 9$ in contrast did not support stable interaction with VEGFR2 (Supplemental Figure S2, A-C); thus, DUF2C5 was defined as the minimum binding region within DUF2 domain of KIF13B that mediated binding to VEGFR2.
Next we determined bioactivities in two-dimensional capillary network formation assay in HUVECs expressing KIF13B truncated mutants or vector controls. HUVECs were infected with lentiviruses encoding FLAG-C2, -C3, $-\mathrm{C} 5,-\mathrm{C} 8,-\mathrm{C} 9$, or vector. In addition, C-terminus region of KIF13B (1528 to 1826 amino acids), which does not bind to bind to VEGFR2, ${ }^{16}$ was used as a negative control. Expression of truncated mutants $\mathrm{C} 2, \mathrm{C} 3$, and $\mathrm{C} 5$, which bind VEGFR2, significantly reduced VEGF-induced network formation, whereas vector-infected control HUVECs and $\mathrm{C}_{\mathrm{T}}$-expressed HUVECs formed capillary networks on Matrigel in the presence of VEGF (Figure 2, B and C). DUF2C8 and C9, which weakly bind VEGFR2, did not significantly reduce network formation (Figure 2, $\mathrm{B}$ and $\mathrm{C}$ ).

To address the role of KIF13B in mediating angiogenesis in vivo, we next performed the Matrigel plug assay in mice ${ }^{16,30}$ using lentivirus expressing truncated mutants of 

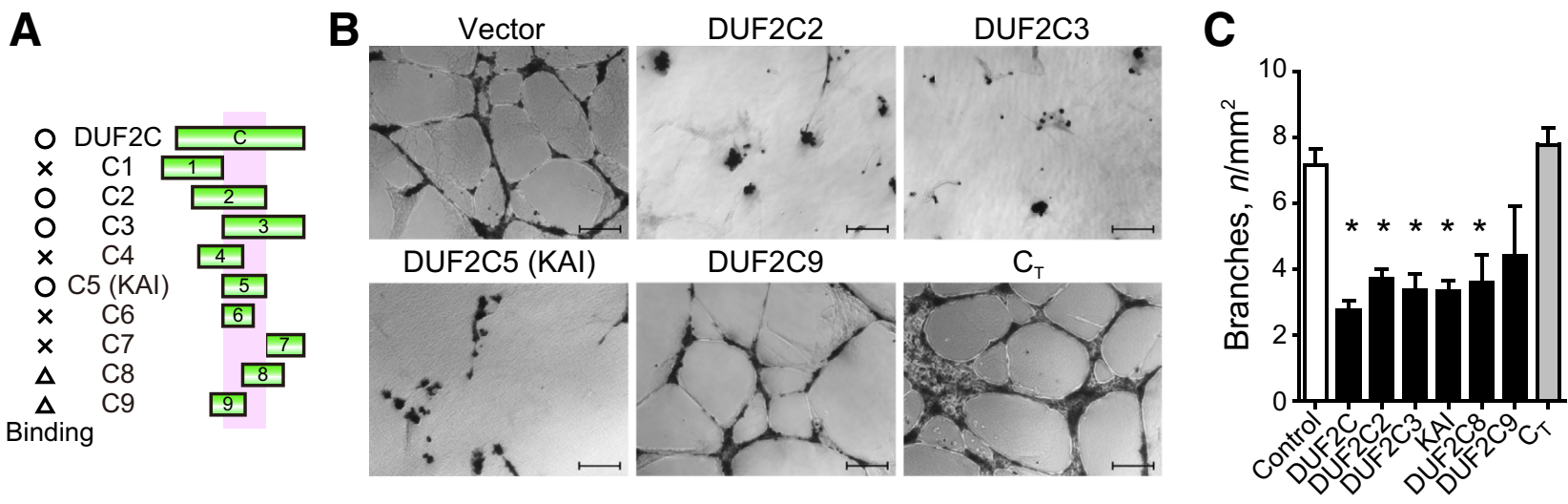

D
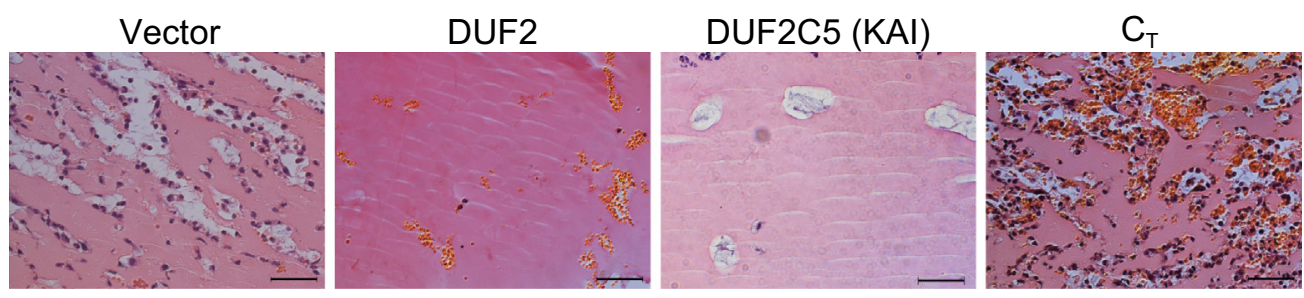

E

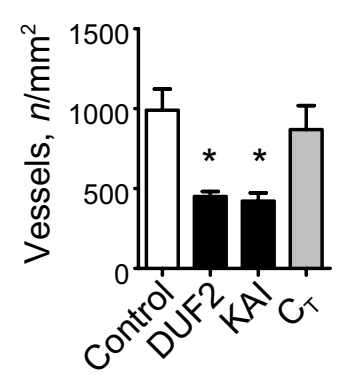

Figure 2 Peptides derived from KIF13B inhibit vascular endothelial growth factor (VEGF)-induced angiogenesis. A: Schematic of the domains of KIF13B and truncated domains used in the study. Domains of unknown function (DUF) 2C1 [1202-1240 amino acids (aa)], C2 (1221-1260 aa), C3 (1241-1281 aa), C4 (1226-1251 aa), C5 (1238-1260 aa), C6 (1238-1254 aa), C7 (1261-1281 aa), C8 (1251-1268 aa), and C9 (1235-1252 aa) were expressed as recombinant proteins in bacteria and tested for binding to vascular endothelial growth factor receptor 2 (VEGFR2) by pull-down assay. 0, $X$, and triangle indicate binding, no binding, and partial or unstable binding, respectively. Binding region is indicated as pink shadow. B and C: In vitro two-dimensional capillary network formation of human primary umbilical vein endothelial cells treated with either control virus or virus encoding truncated mutants of FLAG-KIF13B in Matrigel supplemented with VEGF. $C_{\mathrm{T}}$ (1528-1826 aa) was used as a negative control because it did not bind to VEGFR2. ${ }^{16} \mathbf{D}$ and E: Hematoxylin and eosin staining of Matrigel plug was supplemented with $4.4 \mathrm{nmol} / \mathrm{L}$ VEGF, $50 \mathrm{ng} / \mathrm{mL}$ of basic fibroblast growth factor, $60 \mathrm{U}$ of heparin, and $0.8 \times 10^{8}$ IFU lentivirus (vector control, DUF2, or DUF2C5, or $\left.C_{T}\right)$ and injected s.c. in C57BL6 mice. Data are expressed as means \pm SEM (C and E). $n=15,9,6,6,6,6,6$, and 3 for the groups, respectively $(\mathbf{C}) ; n=4,4,5$, and 5 for vector control, DUF2, DUF2C5, and $C_{T}$, respectively $(\mathrm{E})$. ${ }^{*} P<0.05$ (one-way analysis of variance). Scale bars $=100 \mu \mathrm{m}$ (B and D). IFU, infectious unit; KAI, kinesin-derived angiogenesis inhibitor.

KIF13B (Figure 2, D and E, and Supplemental Figure S2D). High titer of lentivirus was injected with Matrigel s.c. into C57BL mice in the presence of VEGF, bFGF, and heparin. Interestingly, expression of DUF2C5 or full-length DUF2 significantly reduced the number of vessels, whereas vector control or expression of $\mathrm{C}_{\mathrm{T}}$ had no significant effect (Figure 2, D and E, and Supplemental Figure S2D). These results identifying the minimum binding site of KIF13B for VEGFR2 revealed that expression of the minimum binding site inhibited angiogenesis in both the in vitro and in vivo angiogenesis assays. We termed the minimum binding site as a KAI.

\section{Antiangiogenesis Peptide KAI Derived from KIF13B Prevents Tumor Angiogenesis}

Protein BLAST search revealed that KAI did not overlap with any other protein sequence. Using competitive binding assay, we determined whether KAI competed for binding of VEGFR2 with endogenous KIF13B in HUVECs. VEGFR2 coimmunoprecipitated with KIF13B in control cells, whereas preincubation with KAI prevented the interaction of VEGFR2 with KIF13B (Figure 3A). Because interaction of KIF13B and VEGFR2 is necessary for trafficking of VEGFR2 to cell surface, ${ }^{16}$ we next examined the cell surface expression of VEGFR2 in the absence or presence of KAI. Serum-starved HUVECs were preincubated with the peptide $(3,10 \mu \mathrm{mol} / \mathrm{L})$ or PBS control, and then the cells were stimulated with VEGF for the indicated times. Cell surface localized VEGFR2 was stained by antibody against extracellular domain of VEGFR2 as described. ${ }^{16}$ In control cells, VEGFR2 was detected on the cell surface before VEGF stimulation, VEGFR2 disappeared at 1 hour after stimulation, and cell surface pool was restored by the transport of newly synthesized VEGFR2. Initial VEGFR2 cell surface accumulation and internalization, however, were unaffected by preincubation of KAI (data not shown); that is, KAI only prevented the restoration of cell surface VEGFR2 after VEGF stimulation (Figure 3, B and C).

To examine the specificity of KAI, we designed a negative control peptide that does not bind to VEGFR2 (Figure $4 \mathrm{~A}$ ). Because the $\mathrm{C}_{\mathrm{T}}$ region of KIF13B does not 


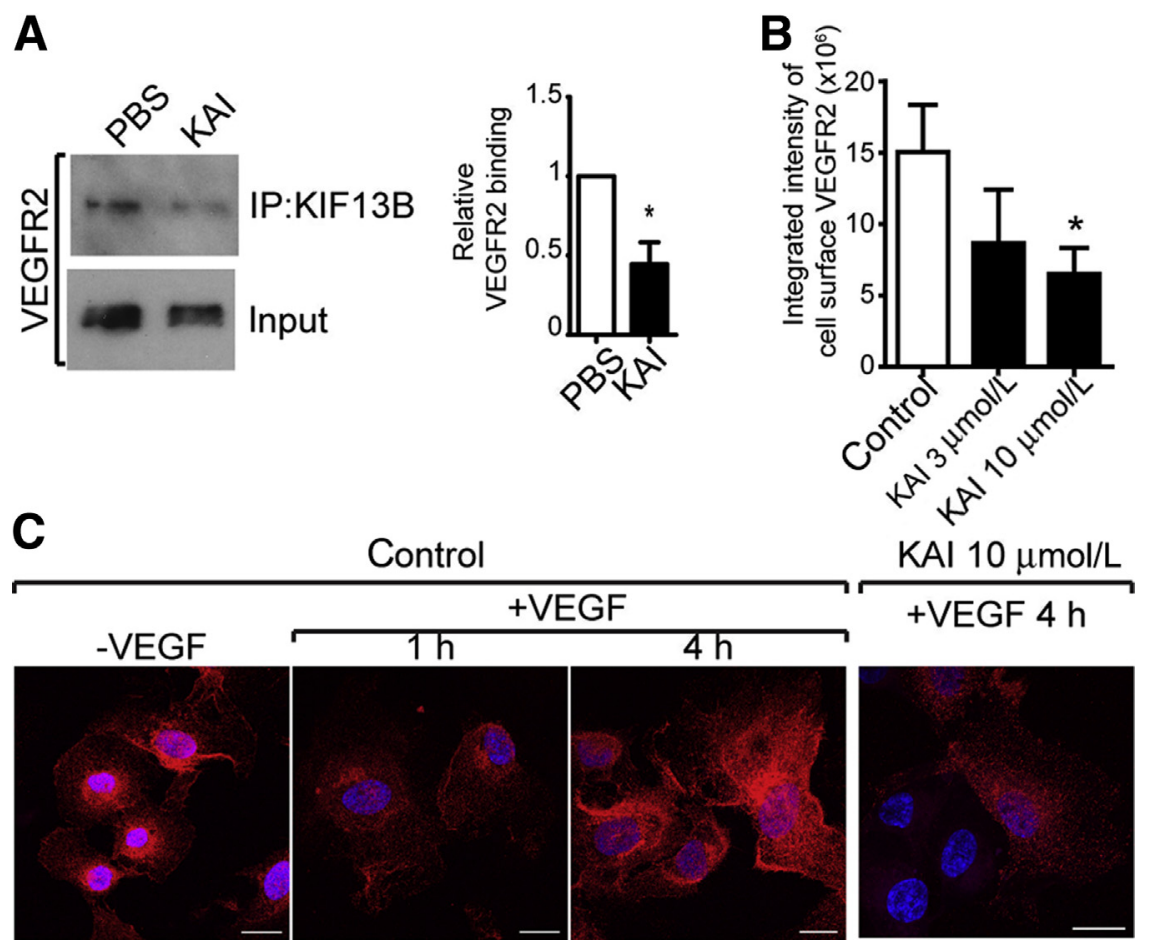

Figure 3 The peptide kinesin-derived angiogenesis inhibitor (KAI) inhibits vascular endothelial growth factor receptor 2 (VEGFR2) trafficking to endothelial cell surface by preventing binding of VEGFR2 cargo to KIF13B. A: Competitive binding assay performed in human primary umbilical vein endothelial cells (HUVECS). HUVECs were preincubated with KAI for 1 hour and stimulated with vascular endothelial growth factor (VEGF) (2.2 $\mathrm{nmol} / \mathrm{L}$ ) for 1 hour. Immunoprecipitation (IP) of VEGFR2 with anti-KIF13B antibody was detected with anti-VEGFR2 antibody. B and C: Effects of peptide on VEGFR2 transport to the cell surface after VEGF (2.2 nmol/L) treatment of HUVECs. Data are expressed as means $\pm \operatorname{SEM}(\mathbf{A}$ and $\mathbf{B}) . n=3$ (A); $n=14,11$, and 23 for control, KAI $3 \mu \mathrm{mol} / \mathrm{L}$, and KAI $10 \mu \mathrm{mol} / \mathrm{L}$, respectively. ${ }^{*} P<0.05$ ( $t$-test and one-way analysis of variance). Scale bars $=$ $50 \mu \mathrm{m}(\mathbf{C})$. bind VEGFR2, ${ }^{16}$ we synthesized a 23 -amino acid peptide termed CT23 (1650- to 1672 amino acids) from the $\mathrm{C}_{\mathrm{T}}$ region (Figure 4A). We first confirmed peptide interaction with VEGFR2 using the pull-down assay. Biotin-KAI peptide immobilized on streptavidin beads pulled down VEGFR2 from HUVEC lysates, whereas beads alone or CT23 on beads did not interact with VEGFR2 (Figure 4B). Next, we determined EC uptake of the peptides. Both KAI and CT23 were designed to have high $\mathrm{pI}(\mathrm{pH}$ at which the molecules carry no net charge), pI 12.1 and 10.4, respectively; thus, in neutral $\mathrm{pH}$, both peptides have a positive charge important for cellular uptake. ${ }^{29}$ We tested the cellular uptake of KAI and CT23 in HUVECs. KAI and CT23 were synthesized with biotin-tag and visualized using streptavidin-Alexa418. After incubation with peptides for 2 hours, HUVECs having internalized peptides were quantified by flow cytometry (Supplemental Figure S3A).

Next, we tested the effects of KAI on VEGF-induced proliferation by the WST-1 assay (Supplemental Figure S3B). KAI inhibited VEGF-induced proliferation at concentrations of 3 and $10 \mu \mathrm{mol} / \mathrm{L}$, whereas CT23 at 3 $\mu \mathrm{mol} / \mathrm{L}$ had no effect. VEGF-induced increase in endothelial permeability was also determined using confluent HUVEC monolayers (Supplemental Figure S3C). KAI inhibited VEGF-induced increase in permeability, whereas CT23 did not. Next, we determined HUVEC viability after incubation with $\operatorname{KAI}(1,3,10 \mu \mathrm{mol} / \mathrm{L})$ for 24 hours using WST-1 assay. Even the highest KAI concentration of $10 \mu \mathrm{mol} / \mathrm{L}$ failed to alter cell viability (Supplemental Figure S3D). After incubation with $\mathrm{KAI}(10 \mu \mathrm{mol} / \mathrm{L})$, PBS vehicle, or TNF- $\alpha(50 \mathrm{ng} /$
$\mathrm{mL}$ ), we observed that only TNF- $\alpha$, a positive control, induced apoptosis (Supplemental Figure S3, E and F).

EC migration in response to KAI was also determined using the scratch wound healing assay. Control HUVECs (PBS vehicle or CT23) migrated and closed the wound in response to VEGF; however, VEGF-induced migration of HUVECs was markedly delayed in the presence of KAI (Figure 4C). We also tested using the Transwell migration assay whether the inhibitory effect of KAI on EC migration was specific to VEGF. HUVECs were placed in the Transwell chamber with or without KAI or CT23. KAI inhibited VEGF-induced EC migration but did not alter S1P- or bFGF-induced migration (Figure 4D). PBS and CT23 had no effect on EC migration induced by all stimuli.

We then determined changes in VEGF-induced network formation assay in Matrigel plugs. KAI prevented VEGFinduced network formation in a concentration-dependent manner, whereas CT23 had no effect (Figure 5A). VEGFinduced EC sprouting was also determined by 3dimensional culture of HUVECs on beads. ${ }^{26}$ Control cells and CT23-treated cells exhibited formation of long sprouts and multiple branching points, whereas KAI treatment markedly reduced the number of branching point structures and sprouts per bead (Figure 5, C-E).

To determine whether VEGFR2 trafficking regulated angiogenesis an in vivo cancer model, we used the tumor implantation mouse model. ${ }^{31}$ Studies were made using lung carcinoma because it depends on angiogenesis for tumor growth. ${ }^{31}$ Mice with severe combined immunodeficiency xenografted with human lung carcinoma H460 s.c. received 
A

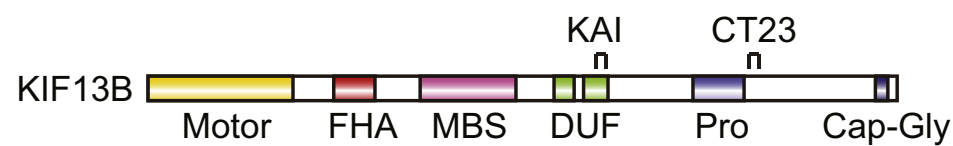

B

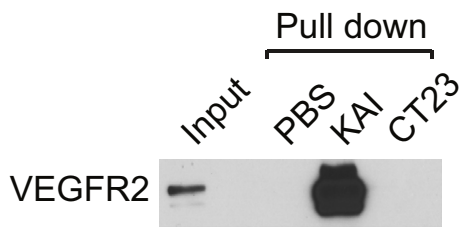

C

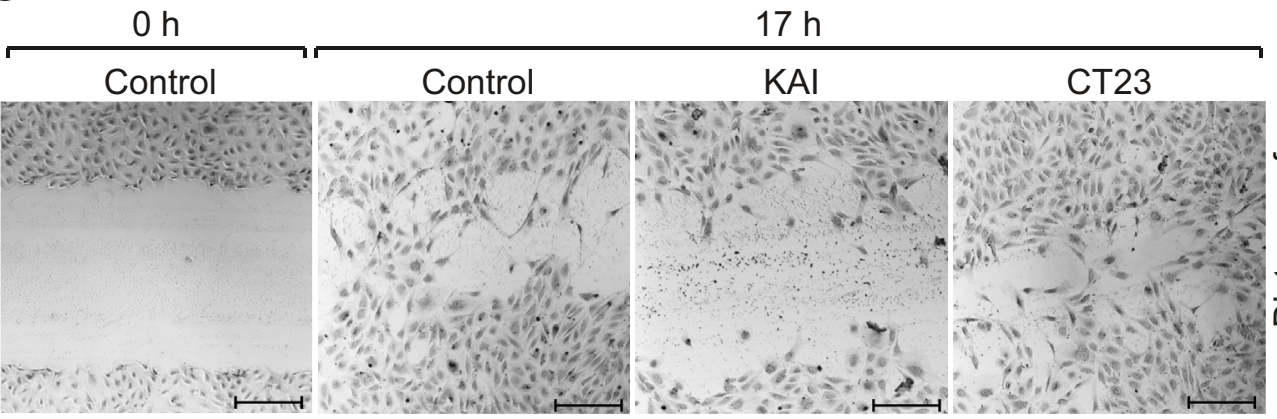

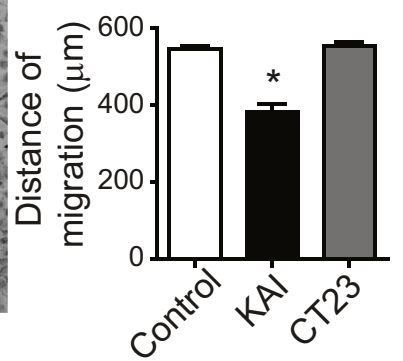

D
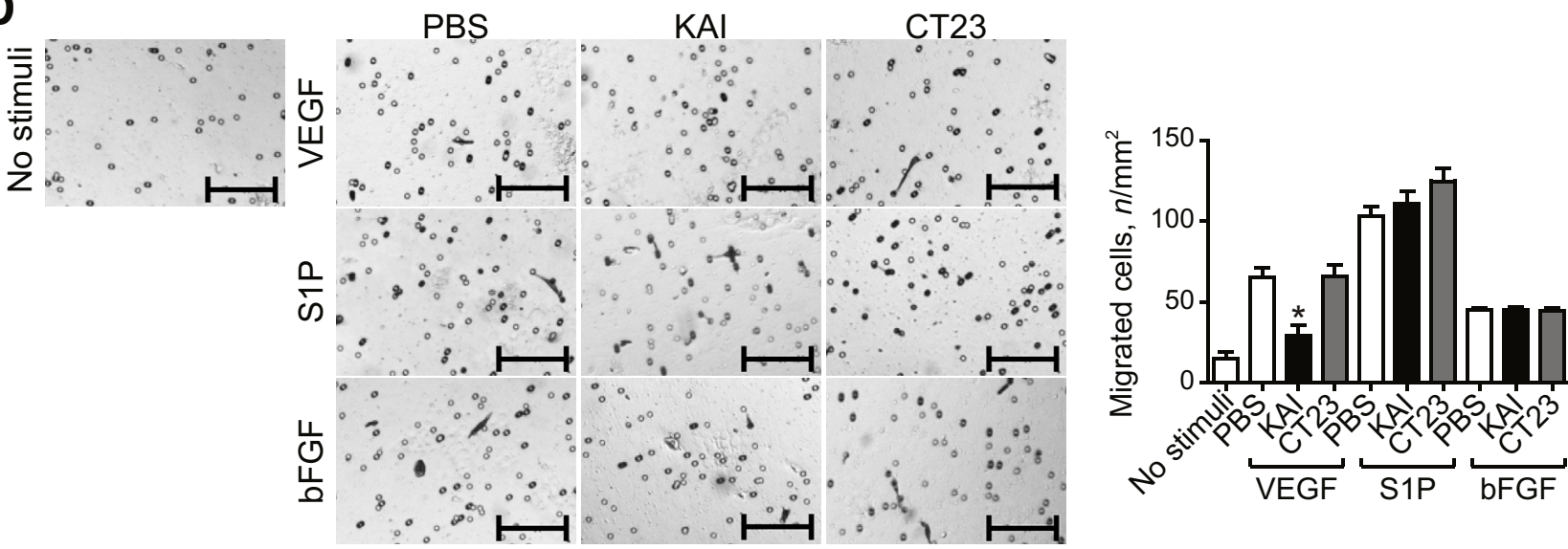

Figure 4 Kinesin-derived angiogenesis inhibitor (KAI) inhibits vascular endothelial growth factor (VEGF)-induced endothelial cell (EC) migration. A: Schematic of domains of KIF13B and synthesized peptides used in the study. B: Pull-down assay for interaction of peptide KAI to vascular endothelial growth factor receptor 2 (VEGFR2), whereas CT23 control peptide did not interact with VEGFR2. C: VEGF-mediated migration of human primary umbilical vein endothelial cells (HUVECs) determined by wound healing scratch assay ${ }^{16}$ in the presence of $2.2 \mathrm{nmol} / \mathrm{L}$ VEGF with or without KAI $(1 \mu \mathrm{mol} / \mathrm{L})$. At indicated times, cells were fixed and stained with hematoxylin. D: Specificity of inhibitory effect of KAI determined by EC migration induced by different stimuli in Transwell migration assay as described. ${ }^{27}$ HUVECs migrated toward $2.2 \mathrm{nmol} / \mathrm{L}$ VEGF, $50 \mathrm{ng} / \mathrm{mL}$ basic fibroblast growth factor (bFGF), or $1 \mu$ mol/L sphingosine-1-phosphate (S1P) were visualized by hematoxylin staining, and number of migrated cells were counted. Data are expressed as means \pm SEM (C and $\mathbf{D}) . n=3(\mathbf{C}$ and $\mathbf{D})$. ${ }^{\star} P<0.05$ (oneway analysis of variance and Bonferroni multiple comparisons test). Scale bars $=200 \mu \mathrm{m}$ (C and D). Cap-Gly, cytoskeleton-associated protein glycine-rich; FHA, forkhead associated; GST, glutathione S transferase; MBS, membrane-associated guanylate kinase-binding stalk; pro, proline rich; S, oligopeptide derived from RNase A (alias S-tag).

either PBS or peptide KAI $(10 \mathrm{mg} / \mathrm{kg})$ by i.v. injection 3 times per week; this dosage is based on the in vitro testing dose regimen described by us (Figures 4 and 5). KAI inhibited tumor growth, whereas PBS-treated control tumor continued to grow (Figure 6A). Blood vessels in tumor visualized by immunohistochemistry using anti-VEGFR2 and anti-von Willebrand factor antibodies revealed extensive vascularity in PBS-treated control tumor, whereas KAI treatment significantly decreased tumor vessel number (Figure 6, B and C). However, KAI did not directly affect viability of the cancer cells (data not shown). TUNELpositive tumor cell number, however, was increased in KAI-treated mice (Figure 6D).
To address whether KAI also reduced angiogenesis in another model, we tested effects in the model of postnatal retina angiogenesis. The peptides KAI or CT23 were injected daily from postnatal day 0 to day 4 , and retina angiogenesis was determined at day 6 . KAI in contrast to the antiangiogenesis effect in tumors had no effect on developmental angiogenesis in retina (Supplemental Figure S4).

\section{Discussion}

We previously reported that VEGFR2 binds KIF13B and that expression of the KIF13B-binding domain inhibited 
A

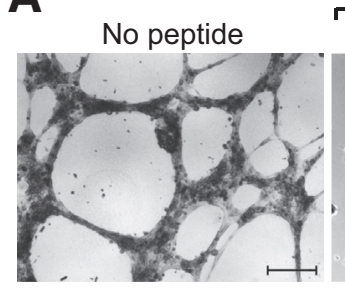

KAI

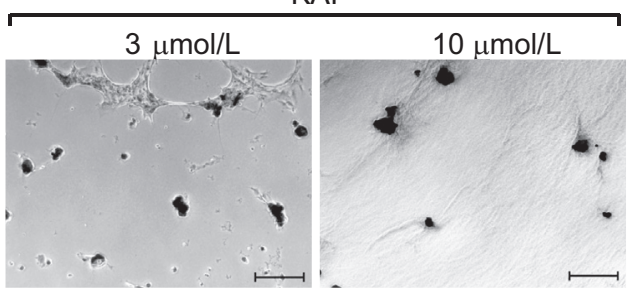

CT23

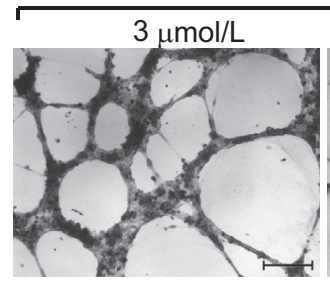

C

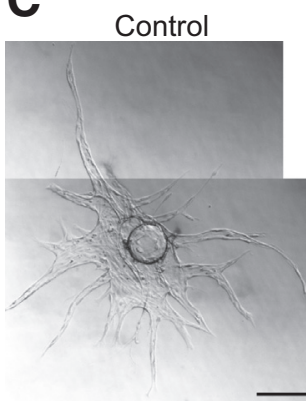

$\mathrm{KAI} 1 \mu \mathrm{mol} / \mathrm{L}$

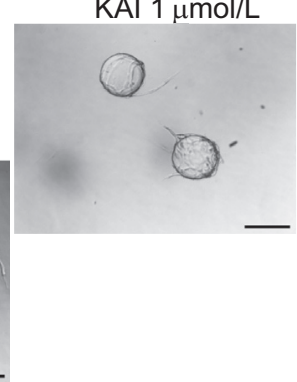

$10 \mu \mathrm{mol} / \mathrm{L}$

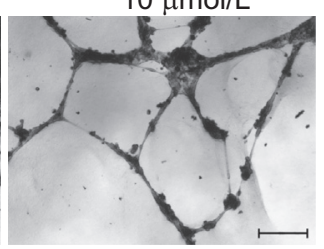

D
B

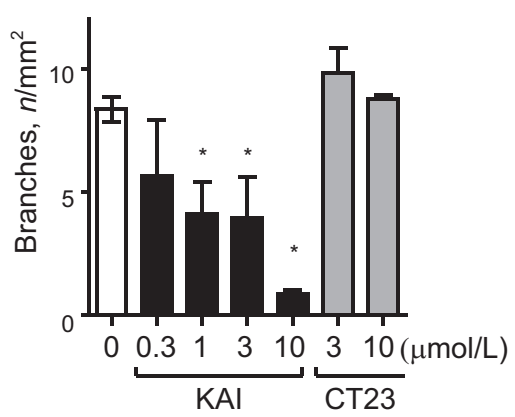

$\mathbf{E}$

Figure 5 Kinesin-derived angiogenesis inhibitor (KAI) inhibits endothelial cell migration, capillary network formation, and sprouting. A and B: In vitro twodimensional capillary network formation. Human primary umbilical vein endothelial cells (HUVECs) were treated with phosphate-buffered saline (PBS) vehicle, synthetic peptides KAI, or CT23 at the indicated doses and tested for capillary network formation in Matrigel in the presence of $2.2 \mathrm{nmol} / \mathrm{L}$ vascular endothelial growth factor (VEGF). C-E: In vitro three-dimensional sprouting assay in fibrin gel supplemented with VEGF (2.2 nmol/L). HUVECs were treated PBS, KAI, or CT23 1 $\mu \mathrm{mol} / \mathrm{L}$ as indicated. Data are expressed as means \pm SEM (B, D, and $\mathbf{E}) . n=10,4,6,4,4,3$, and 3 in the groups, respectively $(\mathbf{B}) ; n=11,9$, and 6 for the control, KAI, and CT23 treated groups, respectively (D and E). ${ }^{\star} P<0.05$ (one-way analysis of variance). Scale bars: $200 \mu \mathrm{m}(\mathbf{A}) ; 50 \mu \mathrm{m}(\mathbf{C})$.

capillary network formation in vitro. ${ }^{16}$ In the present study, we extend these observations and describe a novel 23-amino acid water-soluble and cell-permeable peptide termed KAI derived from the kinesin KIF13B sequence that competes for binding of VEGFR2 to KIF13B and that potently inhibits tumor angiogenesis in a mouse xenograft model. We found that KAI functioned by preventing VEGFR2 trafficking to the plasmalemma and thereby inhibiting EC migration and sprouting.

VEGF/VEGFR2 signaling is an essential target for angiogenesis-dependent diseases, such as cancer and diabetic retinopathy. ${ }^{7,8}$ Anti-VEGF antibody (bevacizumab) and tyrosine kinase inhibitors blocking VEGFR2 activity (sunitinib, pazopanib, sorafenib) are established drugs. ${ }^{7,8}$ Our approach is different because it is based on specifically preventing the trafficking of VEGFR2 to the leading edge of ECs that is mediated by the molecular motor KIF13B. ${ }^{16}$ The underlying premise is that effectiveness of VEGFR2 signaling depends on its localization at the surface of ECs. ${ }^{35-37}$ This is evident in sprouting tip cells in which there is continuous cycling of VEGFR2 from the Golgi to the cell surface pool of
VEGFR2 ${ }^{17}$ VEGFR2 transport to the cell surface is thus needed for each round of ligation by VEGF. ${ }^{16}$ It has been surmised that inhibition of the transport mechanism is a potentially important antiangiogenesis target. ${ }^{14,16,38} \mathrm{We}$ identified a 23-amino acid peptide based on the truncated mutant of KIF13B, which directly binds VEGFR2. This peptide inhibited sprouting angiogenesis solely by blocking transport of VEGFR2 to the EC surface as distinct from antiVEGF antibodies and kinase inhibitors, which decrease VEGF levels and dampen receptor activation. ${ }^{7,8}$

Kinesin inhibitors that target the motor activity of kinesins responsible for mitosis are based on the unique structure of kinesins. Ispinesib, an inhibitor of the kinesin Eg5, ${ }^{39}$ is one such agent. The motor domains of kinesins are, however, highly conserved among kinesin members, whereas cargo binding regions are relatively specific for the cargo. Thus, it is possible to design inhibitors for specific cargoes. We studied KIF13B because it is a nonmitotic kinesin essential for transport of molecules in nondividing mature cells. ${ }^{20}$ These kinesins regulate the trafficking of specific signaling molecules and other cargoes that modify 

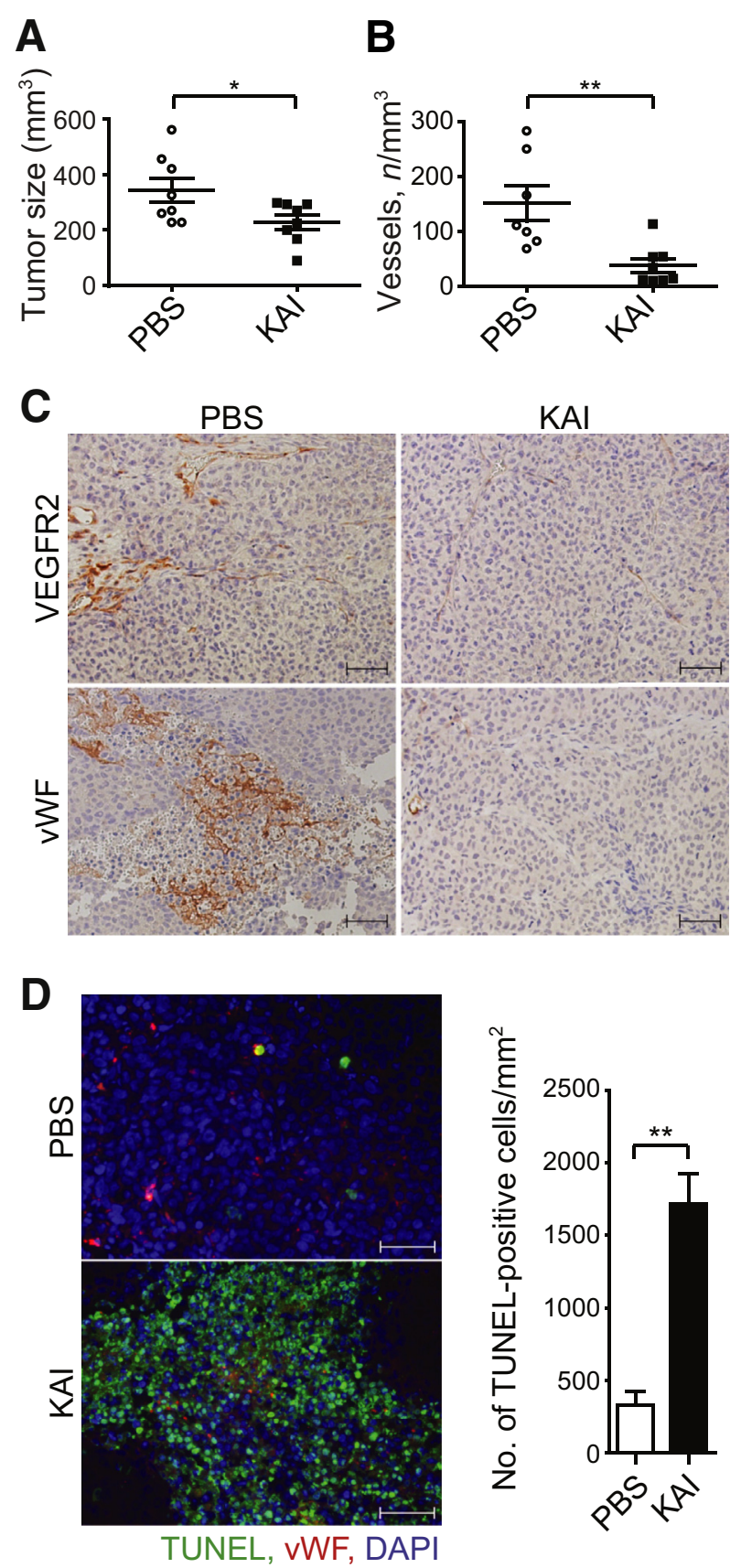

Figure 6 Kinesin-derived angiogenesis inhibitor (KAI) inhibits angiogenesis and tumor growth in vivo. A: Study of human lung carcinoma $\mathrm{H} 460$ xenograft in mice with severe combined immunodeficiency. Mice were treated with KAI (10 $\mathrm{mg} / \mathrm{kg}$ ) or phosphate-buffered saline (PBS), i.v. via tail vein, three times per week, and tumor size is shown in the graph. B and C: Number of vessels in tumor in PBStreated control and peptide-treated tumor. Representative immunohistochemistry of VEGFR2 and von Willebrand factor (VWF) are shown to visualize blood vessels. D: Apoptotic cells visualized with terminal deoxynucleotidyl transferase-mediated dUTP nick-end labeling (TUNEL) assay in tumor tissue treated with PBS vehicle or KAI. Data are expressed as means \pm SEM (A, B, and D). $n=8$ in each group. ${ }^{*} P<0.05,{ }^{* *} P<0.01$ (t-test). Scale bars $=50 \mu \mathrm{m}$ (C and $\left.\mathbf{D}\right)$.

cell function ${ }^{39-43}$ by transporting cargo in the plus or minus end. ${ }^{44}$ Despite the similarity of the motor domain, each nonmitotic kinesin member has a distinct cargo-binding domain that interacts with specific cargo. ${ }^{44}$ KIF13B was known to transport polarity-determination factors, such as $\mathrm{hDlg},{ }^{21} \mathrm{PIP} 3,{ }^{20}$ and VEGFR $2 .{ }^{16}$ In the present study, we identified KAI (1238 to 1260 amino acids of KIF13B) as a minimum binding site for VEGFR2 trafficking in the plus end. We found that it does not overlap with other cargobinding sites for PIP3 and hDlg. We also found that KAI inhibited VEGFR2 trafficking to cell surface and in turn mitigated angiogenesis. This property of KAI was specific for VEGF-induced EC sprouting because KAI did not affect EC migration induced by SIP or bFGF.

KAI was designed by defining the minimum binding site for VEGFR2. KAI is cationic based on its amino acid composition, and the peptide is water soluble $(>10 \mathrm{mg} / \mathrm{mL})$ and cell permeable because of its cationicity. Cellular uptake and delivery of the peptide are likely essential for its effectiveness. ${ }^{29}$ Cationic peptides because of their interaction with negatively charged plasma membrane have the ability to penetrate phospholipid membranes. ${ }^{29}$ Some cationic peptides can also disrupt the membrane ${ }^{45}$; however, we observed that KAI was not itself toxic in ECs, and mice treated i.v. with KAI did not experience any untoward effects. A concern is that KAI may also enter to other cell types in vivo and have off-target effects. siRNA depletion of KIF13B, however, did not affect cell survival or proliferation, ${ }^{16}$ and KIF13B knockout mice had no apparent angiogenic abnormalities. ${ }^{23}$

We also observed that KAI, as opposed to the xenograft cancer model, did not prevent postnatal retinal angiogenesis. The reasons for this are unclear. One possibility is that, in contrast to VEGF/VEGFR2 dependence of angiogenesis in some tumors, ${ }^{46-49}$ postnatal retinal angiogenesis in mice also relies on other factors, such as bFGF and hypoxia, ${ }^{50}$ which may be relatively more important. ${ }^{51}$

In summary, we describe a novel KIF13B-derived peptide selectively inhibiting VEGFR2 binding to KIF13B, which prevents VEGF-induced angiogenesis in tumors by inhibiting VEGFR2 trafficking to the plasma membrane. These results provide the first proof of concept that a specific peptide-reducing cell surface pool of VEGFR2 by blocking KIF13B interaction with VEGFR2 in ECs is a potential strategy for preventing inappropriate angiogenesis and nutrient blood supply in cancer.

\section{Acknowledgments}

We thank Drs. Glenn Marsboom, Kishore Wary, and Masuko Ushio-Fukai for technical advice on the immunohistochmiestry studies; Dr. Tohru Yamada for technical advice regarding in vivo xenograft model and i.v. injection; Yuka Irei for technical assistance; and Dr. Jae-won Shin for critical review.

K.H.Y. and A.B.M. conceived and designed the experiments and wrote the paper. K.H.Y. performed all experiments except for those outlined in Figure 1, D and E, and Supplemental Figure S1, which were performed by H.K. 


\section{Supplemental Data}

Supplemental material for this article can be found at http://dx.doi.org/10.1016/j.ajpath.2016.09.010.

\section{References}

1. Shibuya M, Claesson-Welsh L: Signal transduction by VEGF receptors in regulation of angiogenesis and lymphangiogenesis. Exp Cell Res 2006, 312:549-560

2. Adams RH, Alitalo K: Molecular regulation of angiogenesis and lymphangiogenesis. Nat Rev Mol Cell Biol 2007, 8:464-478

3. Chung AS, Ferrara N: Developmental and pathological angiogenesis. Annu Rev Cell Dev Biol 2011, 27:563-584

4. Folkman J: Angiogenesis: an organizing principle for drug discovery? Nat Rev Drug Discov 2007, 6:273-286

5. Potente M, Gerhardt H, Carmeliet P: Basic and therapeutic aspects of angiogenesis. Cell 2011, 146:873-887

6. Folkman J: Tumor Angiogenesis: therapeutic implications. N Engl J Med 1971, 285:1182-1186

7. Crawford Y, Ferrara N: VEGF inhibition: insights from preclinical and clinical studies. Cell Tissue Res 2009, 335:261-269

8. Ebos JM, Kerbel RS: Antiangiogenic therapy: impact on invasion, disease progression, and metastasis. Nat Rev Clin Oncol 2011, 8: $210-221$

9. Crawford Y, Ferrara N: Tumor and stromal pathways mediating refractoriness/resistance to anti-angiogenic therapies. Trends Pharmacol Sci 2009, 30:624-630

10. Bergers G, Hanahan D: Modes of resistance to anti-angiogenic therapy. Nat Rev Cancer 2008, 8:592-603

11. Chen HX, Cleck JN: Adverse effects of anticancer agents that target the VEGF pathway. Nat Rev Clin Oncol 2009, 6:465-477

12. Jain RK, Duda DG, Willett CG, Sahani DV, Zhu AX, Loeffler JS, Batchelor TT, Sorensen AG: Biomarkers of response and resistance to antiangiogenic therapy. Nat Rev Clin Oncol 2009, 6:327-338

13. Ewan LC, Jopling HM, Jia H, Mittar S, Bagherzadeh A, Howell GJ, Walker JH, Zachary IC, Ponnambalam S: Intrinsic tyrosine kinase activity is required for vascular endothelial growth factor receptor 2 ubiquitination, sorting and degradation in endothelial cells. Traffic 2006, 7:1270-1282

14. Manickam V, Tiwari A, Jung JJ, Bhattacharya R, Goel A, Mukhopadhyay D, Choudhury A: Regulation of vascular endothelial growth factor receptor 2 trafficking and angiogenesis by Golgi localized t-SNARE syntaxin 6. Blood 2011, 117:1425-1435

15. Gampel A, Moss L, Jones MC, Brunton V, Norman JC, Mellor H: VEGF regulates the mobilization of VEGFR2/KDR from an intracellular endothelial storage compartment. Blood 2006, 108:2624-2631

16. Yamada KH, Nakajima Y, Geyer M, Wary KK, Ushio-Fukai M, Komarova Y, Malik AB: KIF13B regulates angiogenesis through Golgi to plasma membrane trafficking of VEGFR2. J Cell Sci 2014, $127: 4518-4530$

17. Nakayama M, Nakayama A, van Lessen M, Yamamoto $H$, Hoffmann $S$, Drexler HC, Itoh N, Hirose T, Breier G, Vestweber D, Cooper JA, Ohno S, Kaibuchi K, Adams RH: Spatial regulation of VEGF receptor endocytosis in angiogenesis. Nat Cell Biol 2013, 15:249-260

18. Gerhardt H, Golding M, Fruttiger M, Ruhrberg C, Lundkvist A, Abramsson A, Jeltsch M, Mitchell C, Alitalo K, Shima D, Betsholtz C: VEGF guides angiogenic sprouting utilizing endothelial tip cell filopodia. J Cell Biol 2003, 161:1163-1177

19. Yamada KH, Hanada T, Chishti AH: The effector domain of human Dlg tumor suppressor acts as a switch that relieves autoinhibition of kinesin-3 motor GAKIN/KIF13B. Biochemistry 2007, 46: 10039-10045
20. Horiguchi K, Hanada T, Fukui Y, Chishti AH: Transport of PIP3 by GAKIN, a kinesin-3 family protein, regulates neuronal cell polarity. J Cell Biol 2006, 174:425-436

21. Hanada T, Lin L, Tibaldi EV, Reinherz EL, Chishti AH: GAKIN, a novel kinesin-like protein associates with the human homologue of the Drosophila discs large tumor suppressor in T lymphocytes. J Biol Chem 2000, 275:28774-28784

22. Soppina V, Norris SR, Dizaji AS, Kortus M, Veatch S, Peckham M, Verhey KJ: Dimerization of mammalian kinesin-3 motors results in superprocessive motion. Proc Natl Acad Sci U S A 2014, 111: $5562-5567$

23. Kanai Y, Wang D, Hirokawa N: KIF13B enhances the endocytosis of LRP1 by recruiting LRP1 to caveolae. J Cell Biol 2014, 204: 395-408

24. Tarbashevich K, Dzementsei A, Pieler T: A novel function for KIF13B in germ cell migration. Dev Biol 2011, 349:169-178

25. Kang H, Kwak HI, Kaunas R, Bayless KJ: Fluid shear stress and sphingosine 1-phosphate activate calpain to promote membrane type 1 matrix metalloproteinase (MT1-MMP) membrane translocation and endothelial invasion into three-dimensional collagen matrices. J Biol Chem 2011, 286:42017-42026

26. Nakatsu MN, Hughes CC: An optimized three-dimensional in vitro model for the analysis of angiogenesis. Methods Enzymol 2008, 443: 65-82

27. Kaplan N, Urao N, Furuta E, Kim S-J, Razvi M, Nakamura Y, McKinney RD, Poole LB, Fukai T, Ushio-Fukai M: Localized cysteine sulfenic acid formation by vascular endothelial growth factor: role in endothelial cell migration and angiogenesis. Free Radic Res 2011, 45:1124-1135

28. Cai J, Jiang WG, Ahmed A, Boulton M: Vascular endothelial growth factor-induced endothelial cell proliferation is regulated by interaction between VEGFR-2, SH-PTP1 and eNOS. Microvasc Res 2006, 71:20-31

29. Jones SW, Christison R, Bundell K, Voyce CJ, Brockbank SM, Newham P, Lindsay MA: Characterisation of cell-penetrating peptide-mediated peptide delivery. $\mathrm{Br} \mathrm{J}$ Pharmacol 2005, 145: 1093-1102

30. Yang K, Proweller A: Vascular smooth muscle Notch signals regulate endothelial cell sensitivity to angiogenic stimulation. J Biol Chem 2011, 286:13741-13753

31. Eklund L, Bry M, Alitalo K: Mouse models for studying angiogenesis and lymphangiogenesis in cancer. Mol Oncol 2013, 7:259-282

32. Yamada T, Goto M, Punj V, Zaborina O, Chen ML, Kimbara K, Majumdar D, Cunningham E, Das Gupta TK, Chakrabarty AM: Bacterial redox protein azurin, tumor suppressor protein $\mathrm{p} 53$, and regression of cancer. Proc Natl Acad Sci U S A 2002, 99: 14098-14103

33. Chavala SH, Kim Y, Tudisco L, Cicatiello V, Milde T, Kerur N, Claros N, Yanni S, Guaiquil VH, Hauswirth WW, Penn JS, Rafii S, De Falco S, Lee TC, Ambati J: Retinal angiogenesis suppression through small molecule activation of p53. J Clin Invest 2013, 123 $4170-4181$

34. Pitulescu ME, Schmidt I, Benedito R, Adams RH: Inducible gene targeting in the neonatal vasculature and analysis of retinal angiogenesis in mice. Nat Protoc 2010, 5:1518-1534

35. Carmeliet P, Jain RK: Molecular mechanisms and clinical applications of angiogenesis. Nature 2011, 473:298-307

36. Simons M: An inside view: VEGF receptor trafficking and signaling Physiology (Bethesda) 2012, 27:213-222

37. Domigan CK, Ziyad S, Iruela-Arispe ML: Canonical and noncanonical vascular endothelial growth factor pathways: new developments in biology and signal transduction. Arterioscler Thromb Vasc Biol 2015, 35:30-39

38. Lanahan AA, Hermans K, Claes F, Kerley-Hamilton JS, Zhuang ZW, Giordano FJ, Carmeliet P, Simons M: VEGF receptor 2 endocytic trafficking regulates arterial morphogenesis. Dev Cell 2010, 18: $713-724$ 
39. Rath O, Kozielski F: Kinesins and cancer. Nat Rev Cancer 2012, 12 : 527-539

40. Li R, Gundersen GG: Beyond polymer polarity: how the cytoskeleton builds a polarized cell. Nat Rev Mol Cell Biol 2008, 9: $860-873$

41. Arimura N, Kaibuchi K: Neuronal polarity: from extracellular signals to intracellular mechanisms. Nat Rev Neurosci 2007, 8:194-205

42. Bornens M: Organelle positioning and cell polarity. Nat Rev Mol Cell Biol 2008, 9:874-886

43. Rodriguez-Boulan E, Macara IG: Organization and execution of the epithelial polarity programme. Nat Rev Mol Cell Biol 2014, 15: $225-242$

44. Hirokawa N, Noda Y, Tanaka Y, Niwa S: Kinesin superfamily motor proteins and intracellular transport. Nat Rev Mol Cell Biol 2009, 10: $682-696$
45. Madani F, Lindberg S, Langel U, Futaki S, Graslund A: Mechanisms of cellular uptake of cell-penetrating peptides. J Biophys 2011, 2011:414729

46. Zhang Z, Neiva KG, Lingen MW, Ellis LM, Nor JE: VEGF-dependent tumor angiogenesis requires inverse and reciprocal regulation of VEGFR1 and VEGFR2. Cell Death Differ 2009, 17:499-512

47. Matsumoto T, Claesson-Welsh L: VEGF receptor signal transduction. Sci STKE 2001, 2001:re21

48. Nagy JA, Dvorak AM, Dvorak HF: VEGF-A and the induction of pathological angiogenesis. Annu Rev Pathol 2007, 2:251-275

49. McMahon G: VEGF receptor signaling in tumor angiogenesis. Oncologist 2000, 5:3-10

50. Krock BL, Skuli N, Simon MC: Hypoxia-induced angiogenesis: good and evil. Genes Cancer 2011, 2:1117-1133

51. Gariano RF, Gardner TW: Retinal angiogenesis in development and disease. Nature 2004, 438:960-966 